\title{
Asynchronous IC Interconnect Network Design and Implementation Using a Standard ASIC Flow
}

\author{
Bradley R. Quinton \\ Dept. of Electrical and Computer \\ Engineering \\ University of British Columbia \\ bradq@ece.ubc.ca
}

\author{
Mark R. Greenstreet \\ Dept. of Computer Science \\ University of British Columbia \\ mrg@cs.ubc.ca
}

\author{
Steven J.E. Wilton \\ Dept. of Electrical and Computer \\ Engineering \\ University of British Columbia \\ stevew@ece.ubc.ca
}

\begin{abstract}
The implementation of interconnect is becoming a significant challenge in modern IC design. Both synchronous and asynchronous strategies have been suggested to manage this problem. Creating a low skew clock tree for synchronous inter-block pipeline stages is a significant challenge. Asynchronous interconnect does not require a global clock and therefore it has a potential advantage in terms of design effort. This paper presents an asynchronous interconnect design that can be implemented using a standard ASIC flow. This design is considered in the context of a simple interconnect network. The results demonstrate that there is a region of the design space where the implementation provides an advantage over a synchronous interconnect. A detailed comparison of power, area and latency of the two strategies is also provided for a range of IC scenarios.
\end{abstract}

\section{Introduction}

The implementation of interconnect is becoming a significant issue in modern IC design. In older processes, wire delays were not significant when compared to gate delays. This greatly simplified interblock communication since any part of the IC could communicate with any other part without special timing considerations. However, in modern processes, wire delays are significant, and inter-block communication is no longer a simple matter [1]. Without careful design, interconnect may become the critical path on a given IC and limit the overall operation. Repeater insertion has been employed to decrease wire latency and improve performance [2]. However, even optimally placed buffers may not be sufficient and register pipelining is required in some situations [3]. Although register pipelining increases throughput, there is a significant design effort required to implement these synchronous pipeline stages since each individual register requires a low-skew clock. Asynchronous techniques have been suggested as a potential solution to the problem of IC interconnect [4,5]. These asynchronous techniques do not require a global clock. Instead, a local request/acknowledgement protocol is used to coordinated each data transfer.

Although a number of asynchronous protocols and implementations have been explored with the intention of being used for IC interconnect, these implementations require custom designed circuits and user-implemented CAD tools or manual design optimization. These techniques are not feasible for typical ASIC/SoC designs that are implemented with standard cells and commercial CAD tools (often referred to as a standard ASIC flow). In this paper we present an asynchronous interconnect design that can be implemented using only standard cells and optimized using commercially available CAD tools. We explore this design in the context of a simple interconnect network. We show that while the requirements of an ASIC design flow impose limitations on the performance of the asynchronous interconnect there is still a significant portion of the overall design space where an asynchronous interconnect provides an advantage over a synchronous interconnect. In addition, we compare the area, power and latency of synchronous and asynchronous implementations of an interconnect network for a range of IC sizes and constructions. These results are based on a standard ASIC flow and are extracted from silicon-ready placed and routed designs. Finally, we conclude by identifying some relatively simple changes to the standard ASIC flow that could significantly increase the performance and reduce the cost of the asynchronous interconnect.

\section{Related work}

The problem of managing high-speed interconnect has been addressed in a number of ways. For synchronous interconnects, a number of algorithms have been developed which determine the optimal repeater and register insertion locations [3,6]. While these algorithms determine the optimal location of registers to 
pipeline the interconnect and achieve a target clock frequency, they do not address the issues of producing a low-skew clock for each of these registers. High-speed clock tree design remains a significant challenge.

Other research has focused on asynchronous interconnect techniques. These techniques can be classified into two broad categories: 1) bundled-data and 2) delay-insensitive [7]. Bundled-data designs manage a number of bits together, and make use of request/acknowledge signalling which is separate from the data. Because of this separation, these designs require delay-matching between the data bits and the control signalling. In contrast, delay-insensitive designs rely on a data encoding that allows the control signalling to be extracted from the encoded data. This encoding removes the requirement of delay-matching from these designs. While bundled-data techniques tend to be more efficient and higher performance than delayinsensitive techniques, the requirement of delaymatching is not compatible with current commercial CAD tools. Therefore bundled-data techniques were not considered for this work. There are a number of different delay-insensitive designs. These designs primarily differ on the basis of data encoding methods and handshaking protocols. There are many possible data encodings [8], however most research has focused on two of these encodings: 1) dual-rail and 2) 1-of-4. Dual-rail uses a two bit encoding for each data bit, whereas 1-of-4 encoding uses a four-bit code for each two data bits. There are two widely used handshaking protocols: 2-phase and 4-phase. When using 2-phase handshaking the transitions of the control and acknowledgement signals indicate the completion of a transfer. When using 4-phase handshaking the value of the control signals indicate the completion of a transfer. The commercially available Nexus crossbar from Fulcrum Microsystems uses a 1-of-4, 4-phase implementation [5]. Unlike our work the Nexus crossbar is based on custom designed pre-charge domino logic. Work by Bainbridge and Furber has also been based on a 1-of-4, 4-phase approach [4]. Again, unlike our work their work requires a sequential cell that does not exist in normal standard cell libraries (a Celement) and does not make use of circuit optimization from commercial CAD tools. A dual-rail, 2-phase protocol, LEDR, has been described [9]. However this implementation was described in terms of custom dynamic logic, and was not investigated in the context of interconnect with long wires. Finally, Stevens has compared a number of synchronous and asynchronous interconnect implementations using first order models [10]. These first order models do not consider the performance or cost in the context of a standard ASIC flow.

\section{Interconnect network structure}

The consideration of interconnect implementations presented in this paper is motivated by a larger project to develop an interconnect network to connect fixed logic blocks to a programmable logic core (PLC) in large SoCs to facilitate post-silicon debug [11]. While the details of the PLC application are not essential to this paper, there are a number of challenges presented by this application that make it a suitable candidate to explore inter-block interconnect implementations. For the application a large number of block outputs must be connected to a single PLC. Since the blocks are distributed on the IC, the connection to the PLC must span a significant portion of the IC. In addition, the communication between the fixed blocks and the PLC must occur at high-speed in order to allow the IC to operate normally. Furthermore, the various IP blocks on the SoCs will often operate at different clock frequencies, therefore the interconnect must allow simultaneous communication at different clock rates. In this paper we will assume that both the design blocks and the PLC operate synchronously. In the future it may be possible to consider an asynchronous PLC implementation [12]. For the purpose of this paper the important aspects of the network are the multiplexing and transmission of the signals from each of the IC blocks to the PLC. The structure used for this portion of the network is a multiplexed bus (Figure 1). In this paper only the network connecting from the fixed blocks to the PLC is considered. Optionally, a network connecting the PLC outputs to the fixed blocks may be used. It is expected that this de-multiplexing network would have similar structure and interconnect issues.

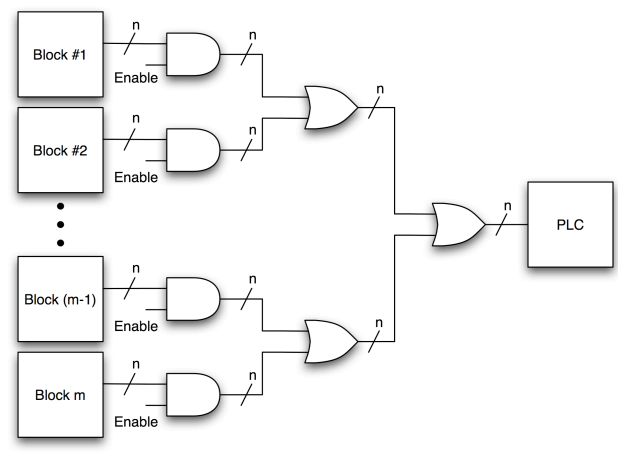

Figure 1 Multiplexed Bus

A multiplexed bus is used for many different IC applications. The multiplexing of signals is very simple and therefore the majority of the challenge of implementing this structure is managing the delay through the network caused by the long distances between the blocks. 


\section{Experimental framework}

\subsection{Target ICs}

In order to investigate the cost and performance of different interconnect network implementations, nine different target ICs were created targeting a TSMC $0.18 \mu \mathrm{m}$ process. Three different die sizes were used: $3830 \times 3830 \mu \mathrm{m}, 8560 \times 8560 \mu \mathrm{m}$, and 12090x12090 $\mu \mathrm{m}$. These die sizes represent total standard cell gate counts of approximately 1 million, 5 million and 10 million respectively. For each die size three different network scenarios were created based on the number of different block partitions on the chip. The three scenarios were: 16 blocks, 64 blocks, and 256 blocks. For simplicity, each of the blocks was set to be of equal size. Signals from each block began at the centre of the block. The location of the PLC (i.e., the network destination) was set to be at the centre of the die (Figure 2). With the exception of the initial signal from each block, the network nodes, wire routing and repeaters were restricted to occur outside the design blocks. The placement guides were used to ensure the placement tool would place network nodes in a location that minimized the distance between each node. To make comparison easier, and shorten tool run times, the width of the multiplexed bus was set to 1 bit wide for all scenarios. This has the side effect of reducing routing congestion, however since the routing was constrained to occur between the design blocks it was determined that there was only a minor optimistic effect on the final timing numbers with respect to a wider bus.

\subsection{CAD tool flow}

Each interconnect network was described using Verilog-HDL. The Verilog description was synthesized with Synopsys Design Compiler (v2004.06) [13]. Synthesis targeted the TSMC $0.18 \mu \mathrm{m}$ 6-layer metal process using the Artisan SAGE-X standard cell library [14]. Functional simulations were done with Cadence Verilog-XL (v05.10) [15]. The cell placement, cell sizing and repeater insertion was performed by Cadence SoC Encounter (v04.10) [15]. Detailed wire routing was performed using Cadence NanoRoute (v04.10) [15]. Parasitic extraction was done using Cadence Fire \& Ice (v04.10). Static timing analysis was performed using Synopsys Primetime (v2004.06) [15]. Verification of pre/post-layout functional equivalency was performed using Synopsys Formality (v2004.06) [15]. Finally, power measurements were done with Synopsys PrimePower (v2003.12) [15].

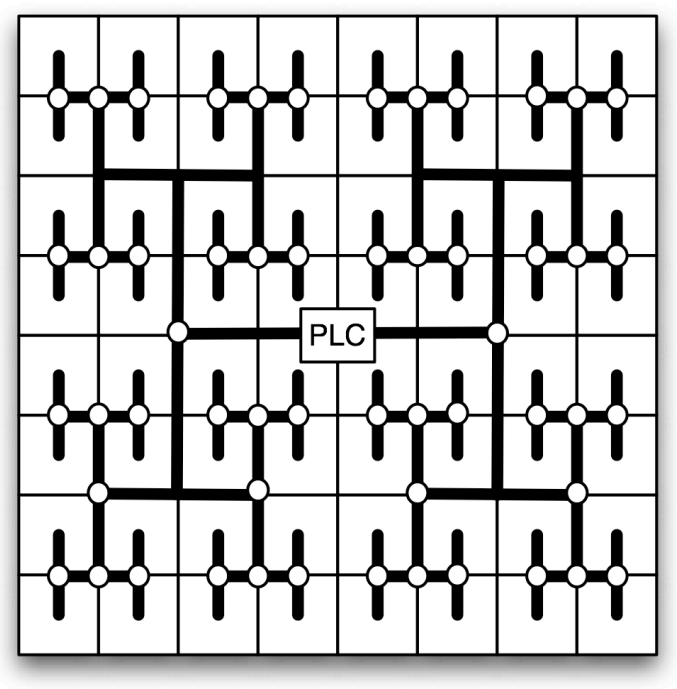

Figure 2 64-Block IC Placement

This tool flow was completely automated using Cshell and tcl scripts (Figure 3). The initial inputs were: a description of size and number of block partitions on the target IC, and the target clock operating frequency. From this initial description both synchronous and asynchronous implementations were created. The outputs of the flow were: a netlist, placement information, a routing description, and area, power and timing details. The run time for the entire flow on a given interconnect ranged from 30 minutes to 3 hours on a $500 \mathrm{MHz}$ UltraSPARC-II processor.

\section{Asynchronous implementation}

\subsection{CAD tool / library limitations}

For the purpose of implementing asynchronous interconnects, there are a number of limitations imposed by commercially available CAD tools. The limitations listed in this section are derived from the specific tool flow outlined in Section 4.2. However, other tools intended for synchronous design impose similar restrictions. The first limitation is that there is no mechanism to ensure that delays are matched on different paths in a circuit. This makes the use of bundled-data interconnects infeasible if the network is large, since each path would require manual consideration. In addition, because of this inability to specify relative delay constraints, there is no automated hazard (glitch) avoidance available. Secondly, these CAD tools will not tolerate combinational feedback paths, and are unable to infer sequential circuits built from combinational gates. Because of this restriction, circuits that create sequential elements that are not 
explicitly defined in the standard cell library cannot be optimized using automatic gate sizing or repeater insertion. Thirdly, for automatic circuit optimization, each path must be referenced to a common global clock. If this reference cannot be made, the tool will simply ignore the path and the delay on that path will not be optimized. Finally, the circuit optimization tools are only designed to insert repeaters to manage wire delays. They do not have the ability to insert sequential elements. For our work, this restriction means that asynchronous pipeline stages can occur only at network nodes.

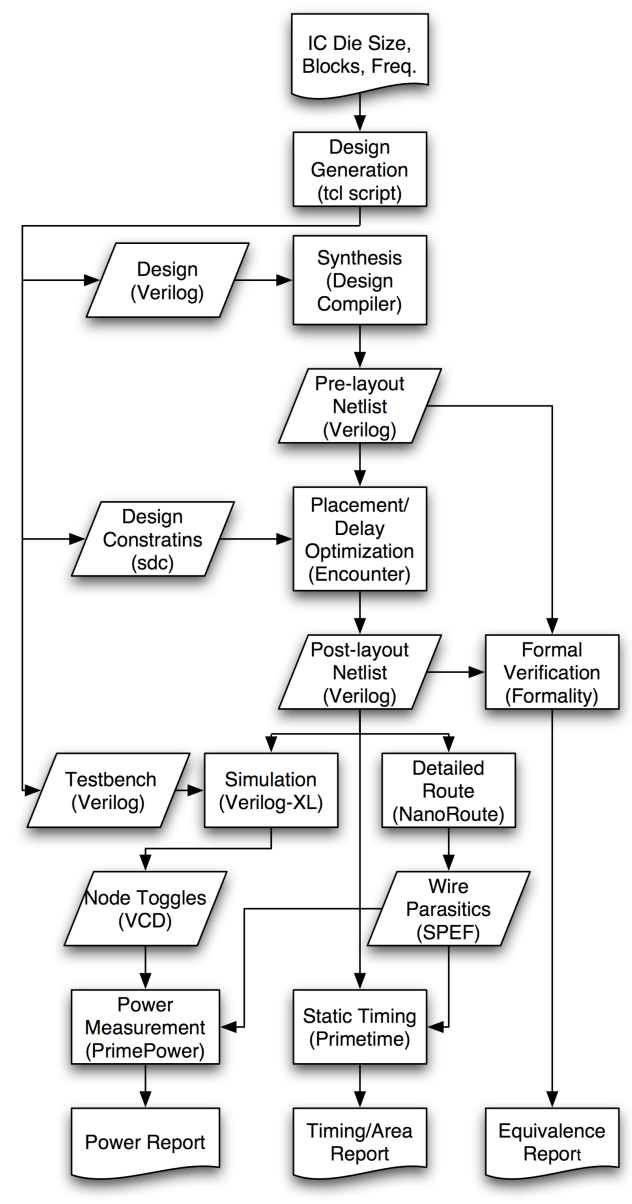

Figure 3 CAD Tool Flow

Standard cell libraries also restrict the implementation of asynchronous interconnects. Since the library is designed for synchronous circuits it lacks certain sequential cells which would be ideal for asynchronous circuits. The most obvious example is the C-element that is used in many asynchronous designs [7].

\subsection{Design}

Our initial designs were based on a 1-of-4, 4-phase protocol since this implementation has been commonly used in previous work on asynchronous interconnect $[4,5]$. However, the throughput restrictions of the 4phase handshaking prevented any of our 4-phase designs from performing at a speed equal to that which could be easily achieved using a synchronous interconnect without any pipelining. With 4-phase signalling, each data transfer requires four traversals of the long wires between the interconnect nodes.

To improve throughput we focused on 2-phase handshaking techniques. We also decided to use a dualrail encoding to minimize the decode logic delay to further improve the throughput. This is the same scheme used by LEDR [10]. In the dual-rail scheme there are two different encodings for each data bit value (Figure 4). This ensures that only one of the wires transitions for each new bit. Based on this encoding it is possible to detect each bit transition with an XOR gate. The value of the XOR gate describes which code exists on the dual-rail. The encoding rules ensure that the code alternates for each new bit. At an asynchronous pipeline stage, the value of the code can be compared to the code that appears in the following stage and the code that represents a new incoming value. The stage accepts a new value only if: 1) the code at the following stage equals the current code, and 2) the incoming code is different from the current code.

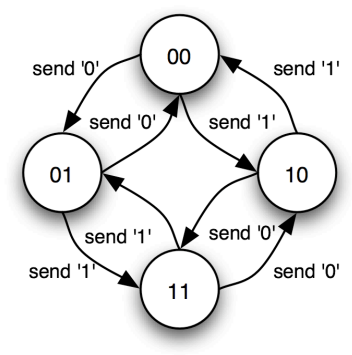

\section{Figure 4 Dual-Rail Encoding}

To meet the requirements of the CAD tool flow we used flip-flops as the sequential elements in the design. (Figure 5a) This use of flip-flops allowed us to specify timing paths that are understandable to circuit optimization tools. The resulting design is quite robust against delay variations. Other than the clock generation circuit, which will be discussed later in this section, there is only one timing relationship that must be ensured: the dual-rail data must reach the ' $d$ ' inputs of the flops before XOR output reaches the clock generation circuitry. This requirement is easily guaranteed by ensuring that the flip-flops and XOR gate 
are placed reasonably close together. We used the 'createRegion' command in SoC Encounter to ensure this relative placement was requirement was met.
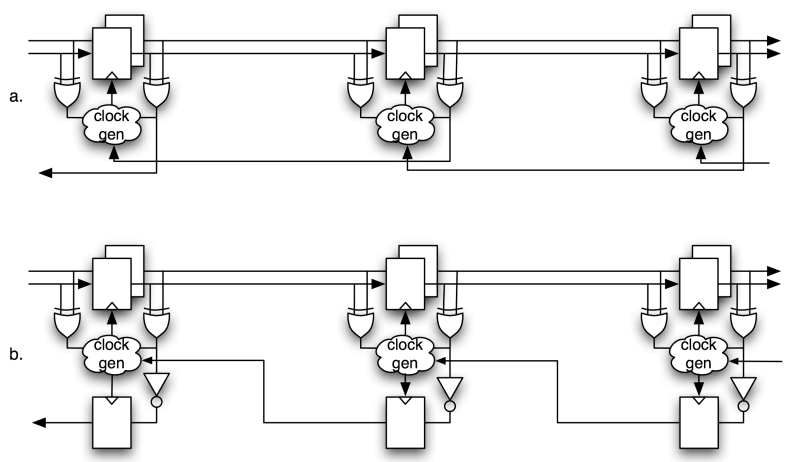

Figure 5 Dual-Rail 2-Phase Implementation

The clock generation circuit was designed using specific discrete gates to avoid hazard issues (Figure 6). In this design a rising clock edge is produced only when the new code is different from the current code and the current code is the same as the next stage code. Therefore there are two possible transitions that generate the rising clock edge: the arrival of a new code, or the arrival of an acknowledgement of the next stage's code (Figure 7). The falling clock edge is always generated by the capture of the new code in the flip-flops (i.e., the generation of a new value for 'current code'). This circuit is robust against delay variations. There is one timing relationship that must be guaranteed: the minimum clock width of the flip-flops must be respected. This requirement is easily met since the falling edge of the clock is not generated until the flip-flops capture a new value and it propagates through the clock generation logic.

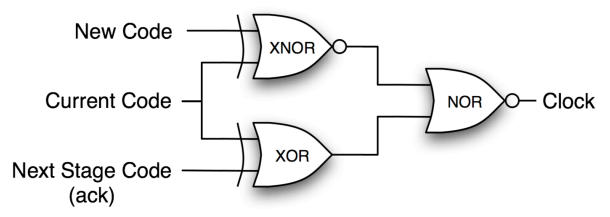

\section{Figure 6 Clock Generation Circuit}

It is possible to further enhance the throughput of the circuit described in Figure 5a. This can be done by pre-computing the acknowledge bit (Figure 5b). This value is then held in an additional flip-flop. By doing this, the delay of one XOR gate is removed from the critical path of the circuit. This modification has the potential to affect the minimum clock width requirement; however if the requirement of placing the flip-flops and clock generation logic together is extended to this new flip-flop, the circuit remains quite safe.

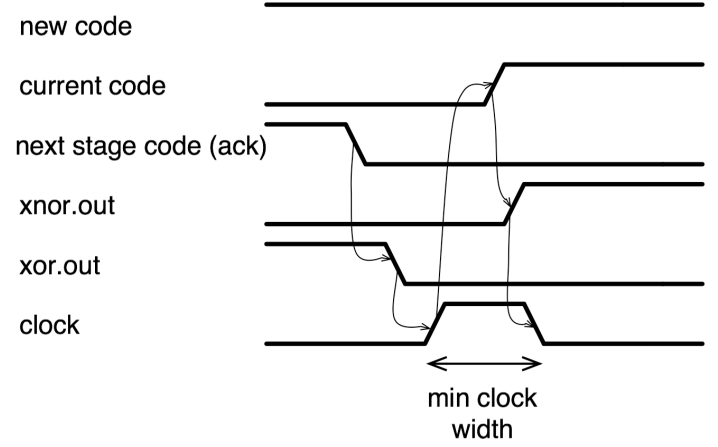

Figure 7 Clock Generation Waveform

\subsection{Delay optimization}

It is possible to perform delay optimization with automatic cell sizing and repeater insertion directly on the circuit described in Section 5.2. However, the descriptions of the clocking relationships can become complicated since the clocks on each flip-flop appear to be unique from the perspective of the CAD tools. Each of these clocks must be related to a common global clock. To simplify this it is possible to make a minor circuit modification immediately before the delay optimization phase. This circuit modification can be corrected once the optimization stage is done. In our flow the functional correctness of this modification is easily verified using a formal verification tool, Synopsys Formality [13]. The circuit modification is done by breaking the wires connecting to the ' $\mathrm{d}$ ' and 'clk' inputs of the flops and then connecting the former clock wire to the ' $\mathrm{d}$ ' input of the flop (Figure 8). This change leaves the 'clk' inputs to the flip-flops open. A 'virtual clock' can then be created and associated with the flip-flop 'clk' inputs. This allows one global clock constraint to be applied to the entire design. It also ensures that the correct timing paths are optimized. Once the optimization is done the 'virtual clock' is removed and the wiring is corrected before the detailed route is performed. Then parasitic extraction and static timing are performed as normal. This wiring modification can be automated in SoC Encounter with the engineering change order (ECO) set of commands. Similar commands exist in other placement tools.

Enabling this automatic delay optimization is a critical part of this tool flow. For instance, in the case of the $12090 \times 12090 \mu \mathrm{m}$ IC with 256 -block partitions, 8856 cells were automatically resized and 232 repeater cells were added to the design. These modifications 
improved the loop time by $12.46 \mathrm{~ns}$ thereby improving throughput by approximately $260 \mathrm{MHz}$. This large change highlights the importance of allowing placement aware delay optimization when using a standard cell based interconnects.
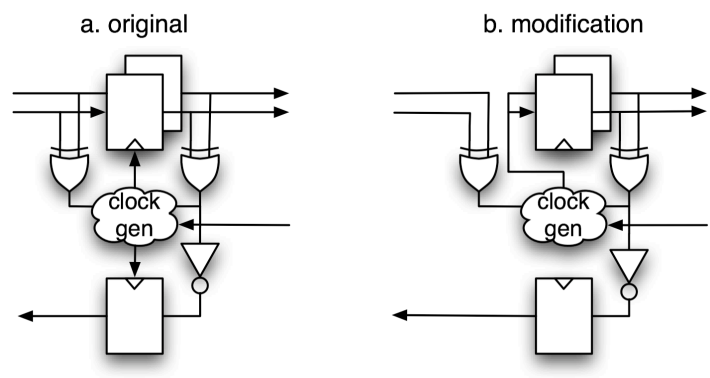

Figure 8 Circuit Modification

\section{Synchronous implementation}

\subsection{CAD tool / library limitations}

The CAD tool flow and standard cell library were intended for synchronous design, therefore there were fewer limitations with respect to implementing the synchronous interconnect. The one significant limitation for this work was that current CAD tools do not support automatic insertion of sequential elements to optimize wire delays. This ability does exist in algorithms described research papers [3] but has not yet been implemented in the tools that we used for this work. Because of this, synchronous pipeline stages were restricted to occurring only at network nodes.

\subsection{Design}

The synchronous design was implemented in a basic manner. Pipeline flops were restricted to the network nodes. Circuits were designed to tolerate a clock skew of 100 ps. In addition a clock uncertainty margin of 100 ps was assumed for all synchronous timing measurements to compensate for jitter and other non-ideal clock tree effects.

\section{Throughput comparison}

The implementations of the interconnect networks described in Section 5 and 6 were performed on the nine target ICs described in section 4.1. Results were generated to compare the implementations for a design scenario where no clock is available for the inter-block interconnect. Due to the absence of a clock the synchronous implementation has no pipeline stages. On the other hand, since the asynchronous implementation does not require a clock to pipeline the asynchronous network is pipelined.

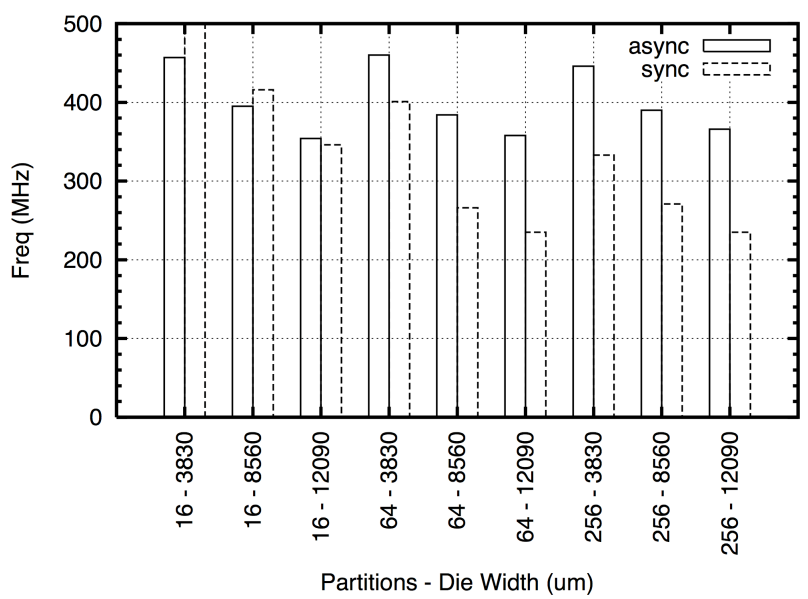

Figure 9 Throughput

The results show that for the larger networks, there is a significant region where the asynchronous interconnect runs faster than the non-pipelined synchronous interconnect (Figure 9). Therefore in this region it is potentially advantageous to use the asynchronous design in order to save the design effort of generating a clock tree for the synchronous pipeline registers. For the particular application that we are considering (connecting a number of IP blocks to a PLC in an SoC) the creation of this global clock tree is particularly difficult since an independent, userselectable, clock is required at the pipeline flops for each different clock frequency used on the SoC. In the more general cases, there is an additional potential advantage of the asynchronous implementation in that the asynchronous implementation will tolerate large clock skew and uncertainty, enabling a globally asynchronous locally synchronous (GALS) implementation strategy [5]. For example the synchronous results in Figure 9 require that there is only 100 ps clock skew and 100 ps clock uncertainty between each of the different blocks on the die. The asynchronous interconnect does not have this requirement.

The asynchronous and synchronous designs have different area, power and latency profiles. These differences will be outlined in detail in Section 8 .

\section{Results}

For each of the nine target ICs described in Section 4.1 we generated synchronous interconnect 
implementations targeting $200 \mathrm{MHz}, 350 \mathrm{MHz}, 500$ $\mathrm{MHz}$, and $750 \mathrm{MHz}$ operation. We also created asynchronous implementations targeting $200 \mathrm{MHz}, 350$ $\mathrm{MHz}$, and $500 \mathrm{MHz}$ operation. For each case the minimum number of pipeline stages was used to meet the target clock frequency. The area, power and latency were determined for each implementation. These values were generated using detailed placed and routed designs. The worst-case library was used for all measurement. The worst-case library represents the slowest process corners with a $1.62 \mathrm{~V}$ supply and die temperature $125^{\circ} \mathrm{C}$. Therefore no manufactured devices would be expected to have timing slower than these values. For simplicity the worst-case library values were also used for power measurements. This allows for a consistent comparison between the two approaches but does not represent the upper bound of the power consumption.

Due to space constraints, only results for the 350 $\mathrm{MHz}$ target are presented for each IC scenario. This is a representative case highlighting the part of the design space where the trade off between using an asynchronous implementation and building clock tree for inter-block pipeline stages occurs for many of the scenarios. The results for area, latency and power are presented below.

The area of both implementations was compared for each of the nine design scenarios (Figure 10). The area reflects the total standard cell area and does not include the wire area. For the synchronous case the area required for a global clock tree was not included. In general the area for the asynchronous case is about eight times larger than the synchronous case. Although relative area difference is quite large, in the context of the overall IC the difference is not that significant. For example the extra area of the asynchronous interconnect for an $8560 \times 8560 \mu \mathrm{m}$ IC with 64 block partitions is approximately $0.09 \%$ per bit.

The latency of both implementations was compared for each of the nine design scenarios (Figure 11). The latency of the asynchronous interconnect is significantly larger than the synchronous case. This latency is a direct result of the delay of the clock generation circuit and the 'clk-q' delay of the flip-flops. We anticipate that the latency could be significantly reduced by adding a small number of cells to the standard cell library. This is discussed as future work in Section 9. Alternatively, there have been proposals for latency-insensitive design techniques [16].

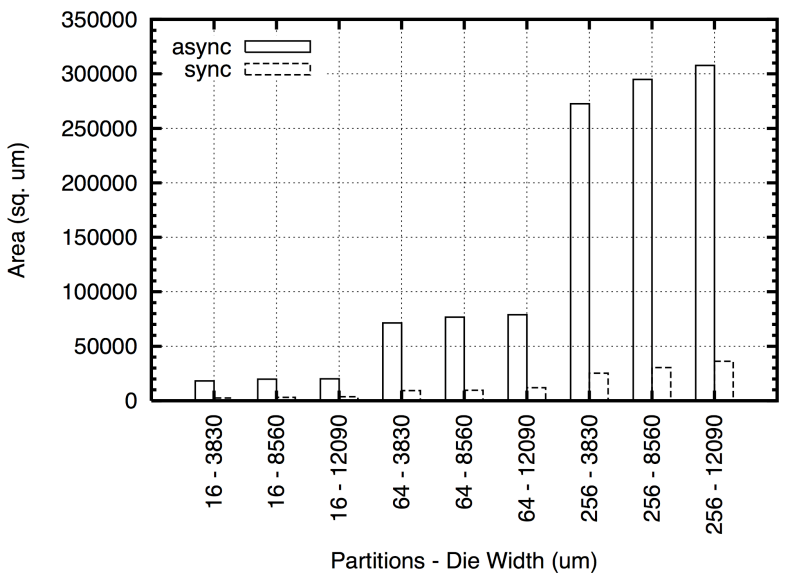

Figure 10 Area

The power consumption of both implementations was compared for each of the nine design scenarios (Figure 12). The results were generated by measuring the power consumption while transmitting a fixed set of 1000 random bits from a single input to the output. To allow for consistent comparisons the results are displayed as the energy per end-to-end transfer. For the synchronous case the power does not include the clock tree distribution power, but does include the power consumed by clocking each individual flip-flop. It is interesting to note that the power of asynchronous case is lower that the synchronous case for some of the larger scenarios. The difference is a result of the fact that in the synchronous case each flip-flop in the design consumes power on each clock cycle. In contrast for the asynchronous case only the flip-flops on the active path consume power.

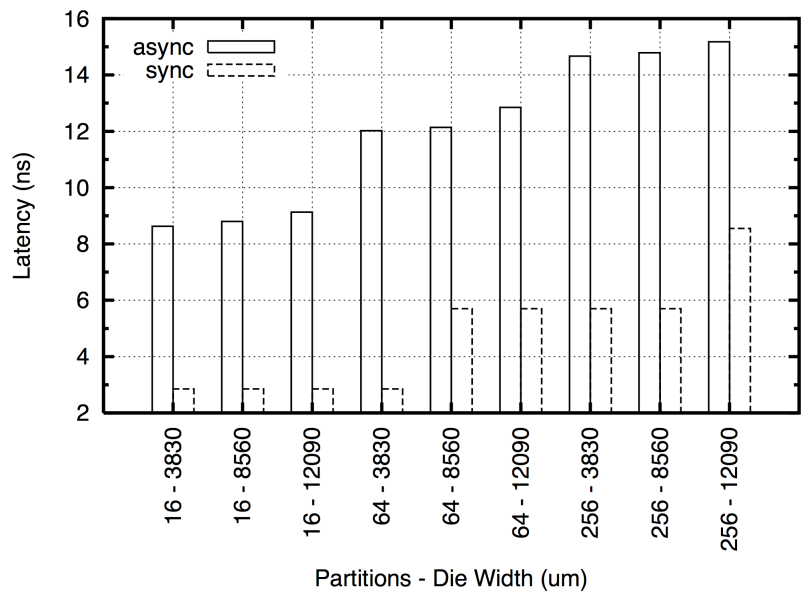

Figure 11 Latency 


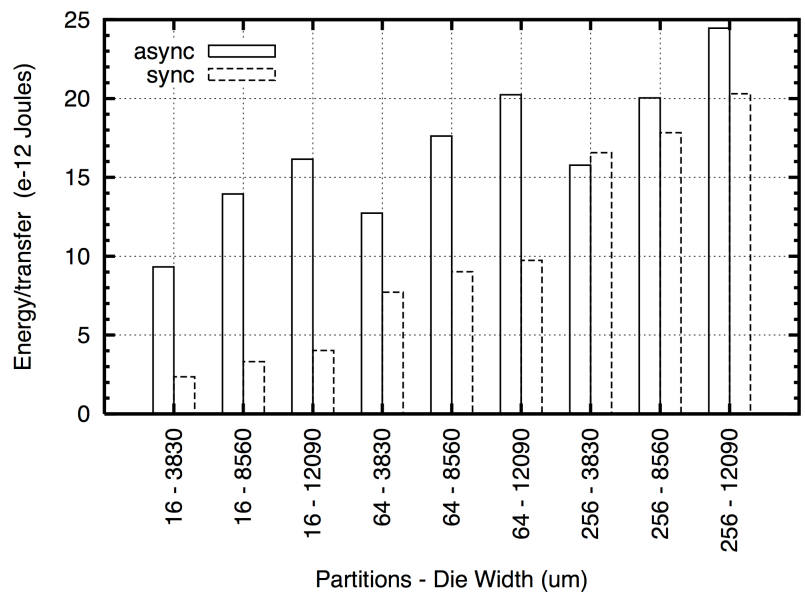

Figure 12 Power

\section{Conclusions and future work}

Asynchronous interconnect is viable in a $0.18 \mu \mathrm{m}$ process using a standard cell library and commercially available CAD tools. We have shown that a 2-phase, dual-rail circuit can be implemented using normal flipflops as sequential elements to allow delay optimization with standard commercial CAD tools. Using our implementation, we demonstrated that there exists a portion of the IC interconnect design space where an asynchronous interconnect provides an advantage over a synchronous interconnect in that it eliminates the need to build complicated clock trees. This interconnect has the added advantage that it tolerates inter-block clock skew. Finally, we have quantified the difference in area, power and latency between our synchronous and asynchronous interconnect implementations. Although the asynchronous design had a much higher area, and a higher power for many cases, this difference is not significant in relation to the total IC design. For many applications the advantage of eliminating global clock tree design and timing closure issues should outweigh this extra area and power.

In completing this work we have been able to identify a number of areas for future work that would involve minor changes to the ASIC flow to improve the performance and reduce the cost of our asynchronous implementation. These changes could be made without altering the basic design flow. The simplest change would be to create a dedicated standard cell for the three input function of the clock generation circuit. There already exists significant number of three input gates in the cell library and therefore this new function would not present a significant alternation of the library. Another library change would be to create a dual input flip-flop specifically for the asynchronous interconnect.
A more significant change would be to modify the delay optimization tools to insert an asynchronous pipeline stage to achieve a target throughput. Although this change is more complicated, a very similar ability would be useful for synchronous designs, therefore we may be able to build this functionality around an existing synchronous tool feature in the future.

\section{References}

[1] R. Ho, K. Mai, and M. Horowitz, "The future of wires", Proc. IEEE, vol. 89, issue 4, April 2001, pp. 490-504.

[2] L.P.P.P. van Ginneken, "Buffer Placement in Distributed RC-Tree Networks for Minimal Elmore Delay", Proc. IEEE Int. Symp. Circuits Syst., 1990, pp. 865-868.

[3] P. Cocchini, "Concurrent Flip-Flop and Repeater Insertion for High Performance Integrated Circuits", Proc. IEEE Int. Conf. Computer-Aided Design, 2002, pp. 268-273.

[4] W.J. Bainbridge and S.B. Furber, "Delay insensitive system-on-chip interconnect using 1-of-4 data encoding", Proc. Int. Symp. on Asynchronous Circuits and Syst., March 2001, pp. 118-126.

[5] A. Lines, "Asynchronous Interconnect For Synchronous SoC Design", IEEE Micro, Vol. 24, No. 1, Jan.-Feb. 2004, pp. 32-41.

[6] C. Lin, and H. Zhou, "Retiming for Wire Pipelining in System-On-Chip", Proc. IEEE Int. Conf. ComputerAided Design, 2003, pp. 215-220.

[7] J. Sparso and S. Furber, Principles of asynchronous circuit design - A system perspective, Kluwer Academic Publishers, 2001.

[8] W.J. Bainbridge, et al, "Delay-Insensitive, Point-to-Point Interconnect using m-of-n Codes", Proc. Int. Symp. on Asychronous Circuits and Syst., 2003.

[9] M. E. Dean, et al, "Efficient Self-timing with LevelEncoded Two-Phase Dual-Rail (LEDR)," Proc. of the 1991 University of California/Santa Cruz Advanced Research in VLSI Conference, MIT Press, March 1991, pp. 55-70.

[10] K.S. Stevens, "Energy and Performance Models for Clocked and Asynchronous Communication", Proc. Int. Symp. on Asynchronous Circuits and Systems, 2003.

[11] B.R. Quinton and S.J.E. Wilton, "Post-Silicon Debug Using Programmable Logic Cores", Submitted to IEEE Int. Conf. on Field-Programmable Technology, 2005.

[12] S. Peng, et al., "Automated Synthesis for Asynchronous FPGAs”, Proc. ACM Int. Conf. on Field Programmable Gate Arrays, Feb. 2005.

[13] Synopsys Inc., http://www.synopsys.com.

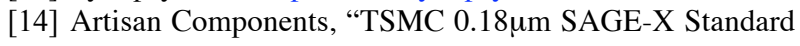
Cell Library", Databook, v4.0, 2002.

[15] Cadence Design Systems, http://www.cadence.com.

[16] L.P. Carloni, and A.L. Sangionvanni-Vincentelli, "Performance Analysis and Optimization of LatencyInsensitive Systems", Proc. Design Automation Conference, 2000. 